\title{
Explosions of O-Ne-Mg cores, the Crab supernova, and subluminous type II-P supernovae
}

\author{
F. S. Kitaura, H.-Th. Janka, and W. Hillebrandt
}

Max-Planck-Institut für Astrophysik, Karl-Schwarzschild-Str. 1, 85741 Garching, Germany

e-mail: thj@mpa-garching.mpg.de

Received 2 December 2005 / Accepted 5 January 2006

\section{ABSTRACT}

We present results of simulations of stellar collapse and explosions in spherical symmetry for progenitor stars in the $8-10 M_{\odot}$ range with an O-Ne-Mg core. The simulations were continued until nearly one second after core bounce and were performed with the PROMETHEUS/VERTEX code with a variable Eddington factor solver for the neutrino transport, including a state-of-the-art treatment of neutrino-matter interactions. Particular effort was made to implement nuclear burning and electron capture rates with sufficient accuracy to ensure a smooth continuation, without transients, from the progenitor evolution to core collapse. Using two different nuclear equations of state (EoSs), a soft version of the Lattimer \& Swesty EoS and the significantly stiffer Wolff \& Hillebrandt EoS, we found no prompt explosions, but instead delayed explosions, powered by neutrino heating and the neutrino-driven baryonic wind which sets in about $200 \mathrm{~ms}$ after bounce. The models eject little nickel $\left(<0.015 M_{\odot}\right)$, explode with an energy of $\gtrsim 0.1 \times 10^{51} \mathrm{erg}$, and leave behind neutron stars (NSs) with a baryonic mass near $1.36 M_{\odot}$. Different from previous models of such explosions, the ejecta during the first second have a proton-to-baryon ratio of $Y_{\mathrm{e}} \gtrsim 0.46$, which suggests a chemical composition that is not in conflict with galactic abundances. No low-entropy matter with $Y_{\mathrm{e}} \ll 0.5$ is ejected. This excludes such explosions as sites of a low-entropy r-process. The low explosion energy and nucleosynthetic implications are compatible with the observed properties of the Crab supernova, and the small nickel mass supports the possibility that our models explain some subluminous type II-P supernovae.

Key words. stars: supernovae: general - stars: supernovae: individual: Crab - neutrinos - hydrodynamics - radiative transfer

\section{Introduction}

Recent observations of subluminous type II-P supernovae (SNe) like 2005cs, 2003gd, 1999br and 1997D, have renewed attention to stars near the lower end of the mass range of core-collapse $\mathrm{SN}$ progenitors, i.e. to stars with about 8-10 $M_{\odot}$, which develop O-Ne-Mg cores. A possible link between both has been suggested because of the low ${ }^{56} \mathrm{Ni}$ and ${ }^{16} \mathrm{O}$ ejecta masses and low progenitor luminosities (e.g., Chugai \& Utrobin 2000; Hendry et al. 2005). However, due to many uncertainties this connection is far from being clear (e.g., Pastorello et al. 2004, 2005; Hamuy 2003; Zampieri et al. 2003, and references therein). Also the Crab Nebula's progenitor was proposed to be in this mass window (Gott et al. 1970; Arnett 1975; Woosley et al. 1980; Hillebrandt 1982). The observed composition of the Crab remnant (small $\mathrm{C}$ and $\mathrm{O}$ abundances, He overabundance) was interpreted as a strong indication that the Crab Nebula comes from a collapsing and exploding progenitor with an O-Ne-Mg core (Davidson et al. 1982; Nomoto et al. 1982; Nomoto 1983).

Moreover, these stars were considered as possible sites for a low entropy r-process (for example, Hillebrandt 1978; Wheeler et al. 1997; Sumiyoshi et al. 2001; Wanajo et al. 2003) based on the assumption that they explode by the prompt bounce-shock mechanism, which Hillebrandt et al. (1984) found to work in a numerical simulation, taking Nomoto's O-Ne-Mg core model (Nomoto 1984, 1987). Such explosions are characterised by the direct propagation of the shock out of the core, the formation of a mass cut, and the continuous acceleration of the material outside of the mass cut to high velocities. They are expected to eject relatively large amounts of neutron-rich matter with low $Y_{\mathrm{e}}(\sim 0.2)$ and low entropies $\left(\sim 10 k_{\mathrm{B}}\right.$ per nucleon). However, several groups could not confirm the viability of the prompt explosion mechanism (Burrows \& Lattimer 1985; Baron et al. 1987; Mayle \& Wilson 1988). Mayle \& Wilson (1988) continued their simulations in the post-bounce phase for a longer time and obtained instead a so-called neutrinodriven, delayed explosion (Bethe \& Wilson 1985) with a low production of ${ }^{56} \mathrm{Ni}$ (approximately $0.002 M_{\odot}$ ) in agreement with subluminous type II-P SNe as mentioned above, but with a vast overproduction of neutron-rich material (at least $0.02 M_{\odot}$ of ejecta with $Y_{\mathrm{e}} \lesssim 0.41$ ). The latter finding is inconsistent with the chemical composition of our galaxy, which allows for no more than $10^{-3} M_{\odot}$ of material with $Y_{\mathrm{e}}<0.42$ being ejected per SN (Hartmann et al. 1985). Moreover, the explosion energies of both studies, around $2 \times 10^{51}$ erg in 
Hillebrandt et al.'s (1984) model and between 0.6 and $1.2 \times$ $10^{51}$ erg in Mayle \& Wilson's (1988) simulations, would be inconsistent with the long plateau phase of the above mentioned subluminous $\mathrm{SNe}$, if their $\mathrm{H}$-envelope masses were $\lesssim 8 M_{\odot}$.

It was suggested that the reason for the discrepant results in the $\mathrm{SN}$ simulations of $\mathrm{O}-\mathrm{Ne}-\mathrm{Mg}$ cores (prompt explosions, delayed explosions, no explosions) could be explained by the different nuclear EoSs used by the groups (Fryer et al. 1999). Having in mind that the different approximations in the neutrino transport in previous calculations introduced additional uncertainties, we revisit this topic with a state-of-the-art neutrino transport treatment together with a careful description of weak interactions and including relevant nuclear burning reactions. We additionally make a comparison of collapse and post-bounce calculations with different nuclear EoSs.

\section{Numerical techniques and input physics}

The transport of neutrinos and antineutrinos of all flavors is done with the energy-dependent solver for the coupled set of moments equations and Boltzmann equation called VERTEX. It is described in detail in Rampp \& Janka (2002). The equations of hydrodynamics are integrated with the Newtonian finitevolume code Prometheus, which uses a third-order, timeexplicit Godunov scheme. This code is a direct implementation of the Piecewise Parabolic Method (PPM), based on a Riemann solver. General relativistic gravity is taken into account approximately by an "effective relativistic potential" according to Marek et al. (2006). Gravitational redshift and time dilation effects are included in the neutrino transport (see Rampp \& Janka 2002).

The code is augmented with improved microphysics as described in Buras et al. (2006). It includes also the improved treatment of electron captures on a large variety of nuclei in nuclear statistical equilibrium (NSE), based on shell model Monte Carlo calculations, as described by Langanke et al. (2003). In addition, electron captures on certain important nuclei in the non-NSE regime, in particular ${ }^{20} \mathrm{Ne}$ and ${ }^{24} \mathrm{Mg}$, are implemented according to Takahara et al. (1989). A simplified treatment of nuclear burning accounts for the main reactions of seven symmetric nuclei ( $\mathrm{He}, \mathrm{C}, \mathrm{O}, \mathrm{Ne}, \mathrm{Mg}, \mathrm{Si}, \mathrm{Ni}$ ). Details about the implemented microphysics will be described in a forthcoming paper (Kitaura et al., in preparation). The nuclear burning reactions considered by Hillebrandt et al. (1984) $\left({ }^{12} \mathrm{C}+{ }^{12} \mathrm{C},{ }^{16} \mathrm{O}+{ }^{16} \mathrm{O},{ }^{12} \mathrm{C}+{ }^{16} \mathrm{O}\right)$ are all included, taking into account different reaction channels.

To describe matter in NSE, we use two different nuclear EoSs in separate simulations, the Wolff \& Hillebrandt (W\&H) EoS (Hillebrandt et al. 1984), which is based on Hartree Fock calculations, and the Lattimer \& Swesty (L\&S) EoS (1991), which is a finite-temperature compressible liquid-drop model and has a compressibility modulus of $180 \mathrm{MeV}$.

This permits us to compare our models with those of Hillebrandt et al. (1984), in which the Wolff and Hillebrandt EoS was used and which produced prompt explosions. The low-temperature and low-density EoS outside of the NSE regime is described by an ideal gas of nuclei and nucleons, electrons, positrons, and photons (Janka 1999). The switch between NSE and non-NSE description was made in a densityand temperature-dependent manner. The initial model is the same as the one used in previous $\mathrm{SN}$ calculations of $\mathrm{O}-\mathrm{Ne}-\mathrm{Mg}$ cores by Hillebrandt et al. (1984) and Mayle \& Wilson (1988). It is a $2.2 M_{\odot}$ He core that corresponds to a progenitor with a main sequence mass of $\sim 8.8 M_{\odot}$ (Nomoto 1984,1987 ). Prior to collapse it has an $\mathrm{O}-\mathrm{Ne}-\mathrm{Mg}$ core with $\sim 1.3 M_{\odot}$, surrounded by a $\mathrm{C}-\mathrm{O}$ shell of about $0.08 M_{\odot}$. We take, however, the initial data at a time when the central density is $\sim 4 \times 10^{10} \mathrm{~g} / \mathrm{cm}^{3}$ and only $\sim 0.1$ solar masses at the center of the core have reached nuclear statistical equilibrium (NSE). This is earlier than the starting configuration taken by other groups, where the core had already a central density of $\sim 3 \times 10^{11} \mathrm{~g} / \mathrm{cm}^{3}$ and where around 0.3 solar masses were in NSE. Our earlier initial model allows us to trace the evolution of the core towards collapse.

We added a helium atmosphere of about $10^{-4} M_{\odot}$ around the $\mathrm{O}-\mathrm{Ne}-\mathrm{Mg}$ and $\mathrm{C}-\mathrm{O}$ core, so that we could move the outer boundary of our Eulerian grid from the core radius of about $1100 \mathrm{~km}$ to $100000 \mathrm{~km}$. For the He-shell profile we adopted a power-law like behaviour of the temperature $\left(T \propto r^{-1}\right)$ from a $10.2 M_{\odot}$ progenitor of A. Heger (private communication), and constructed the density profile by assuming hydrostatic equilibrium, a mass fraction of $100 \% \mathrm{He}$, and using the EoS for the low-density regime. We employ in our simulations a very fine mesh in order to resolve the steep density gradient at the outer boundary of the $\mathrm{C}-\mathrm{O}$ layer, with 1600 nonequidistant zones for the hydrodynamics part. The neutrino transport is done with 235 nonequidistant radial zones.

\section{Results}

Our results show a very smooth transition from the preceding progenitor evolution to core-collapse. Since O-Ne-Mg cores are gravitationally less bound than more massive stellar progenitors and can release more energy due to nuclear burning, a temperature- and density-dependent treatment of all relevant nuclear burning reactions had to be included, combined with a detailed description of the important electron capture rates. Only that ensured that the progenitor evolution continued towards gravitational collapse without numerical transients. We could therefore confirm that the neutrinos produced by electron captures carry away efficiently the energy that is released by nuclear burning (Miyaji et al. 1980; Miyaji \& Nomoto 1987; Hashimoto \& Nomoto 1993). A cruder burning treatment, or omission of the improved electron capture rates on nuclei, can have the consequence that the core expands instead of collapsing to a NS, as we verified in test calculations. Figure 1 shows how the mass shells in the inner region during the first milliseconds start contracting towards the center. The collapse of the core proceeds to higher central densities and when the density of nuclear matter is reached, the EoS "stiffens", and the inner homologous core bounces. The supersonically falling outer layers collide with this central core and a hydrodynamic shock is formed as can be seen in Fig. 1, where this discontinuity in the fluid flow becomes visible by sharp kinks of the mass shell trajectories. This happens after $59 \mathrm{~ms}$ of collapse for the calculation with the W\&H EoS and after $78 \mathrm{~ms}$ in the calculation with the softer L\&S EoS. The shock formation radius is at 


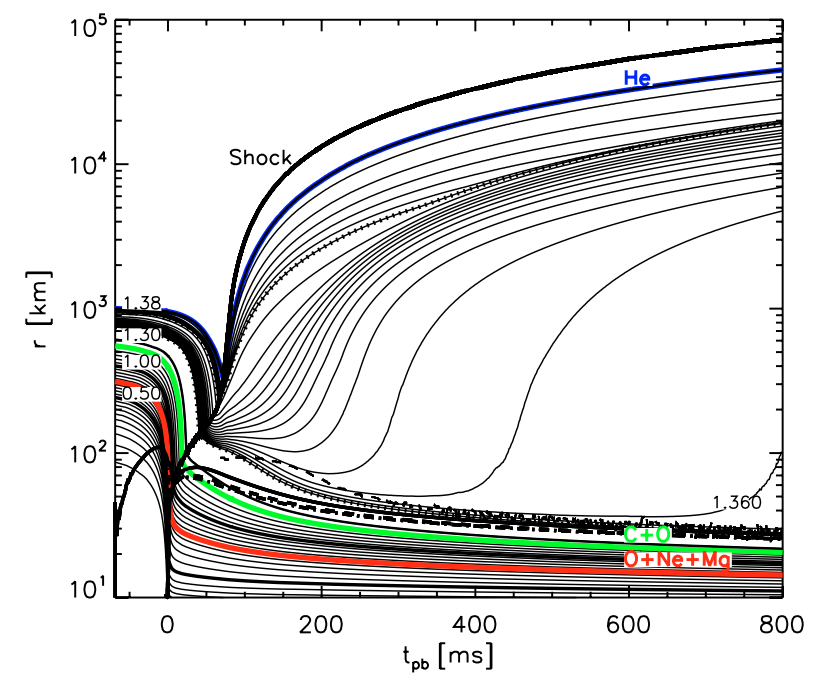

Fig. 1. Mass trajectories for the simulation with the W\&H EoS as a function of post-bounce time $\left(t_{\mathrm{pb}}\right)$. Also plotted: shock position (thick solid line starting at time zero and rising to the upper right corner), gain radius (thin dashed line), and neutrinospheres ( $v_{\mathrm{e}}$ : thick solid; $\bar{v}_{\mathrm{e}}$ : thick dashed; $v_{\mu}, \bar{v}_{\mu}, v_{\tau}, \bar{v}_{\tau}$ : thick dash-dotted). In addition, the composition interfaces are plotted with different bold, labelled lines: the inner boundaries of the $\mathrm{O}-\mathrm{Ne}-\mathrm{Mg}$ layer at $\sim 0.77 M_{\odot}$, of the $\mathrm{C}-\mathrm{O}$ layer at $\sim 1.26 M_{\odot}$, and of the He layer at $1.3769 M_{\odot}$. The two dotted lines represent the mass shells where the mass spacing between the plotted trajectories changes. An equidistant spacing of $5 \times 10^{-2} M_{\odot}$ was chosen up to $1.3579 M_{\odot}$, between that value and $1.3765 M_{\odot}$ it was $1.3 \times 10^{-3} M_{\odot}$, and $8 \times 10^{-5} M_{\odot}$ outside.

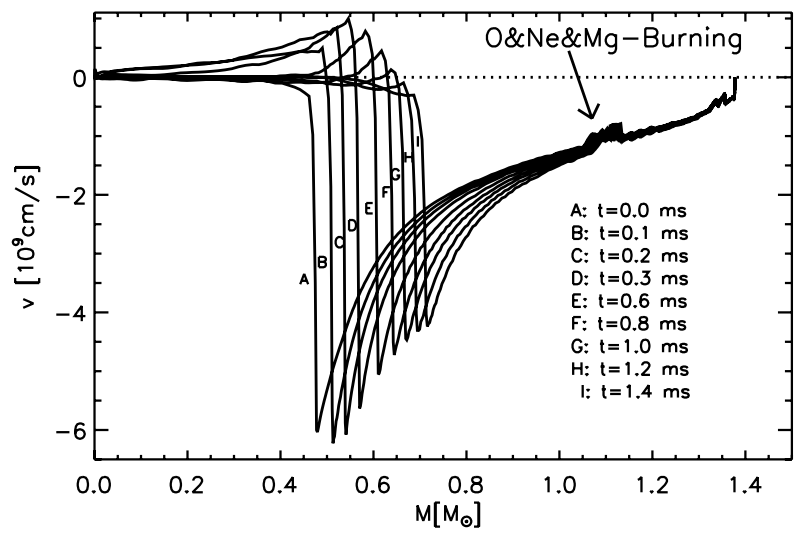

Fig. 2. Velocity profiles vs. enclosed mass at different times for the model with the W\&H EoS. Times are normalized to core bounce.

$1.40 \times 10^{6} \mathrm{~cm}$, corresponding to an enclosed mass of $0.475 M_{\odot}$ in the former case, and at $1.15 \times 10^{6} \mathrm{~cm}$ with an enclosed mass of $0.425 M_{\odot}$ in the second model. This so-called prompt bounce shock produces initially positive velocities in the postshock matter. However, the energy of the shock is insufficient to cause a prompt explosion, and the photodisintegration of nuclei consumes such amounts of energy that the shock is quickly damped and that within only $\sim 1.2 \mathrm{~ms}$ after shock formation the velocities are negative everywhere (see Fig. 2 for the case of the W\&H EoS). Therefore, the prompt shock mechanism fails, independent of the employed nuclear EoS.

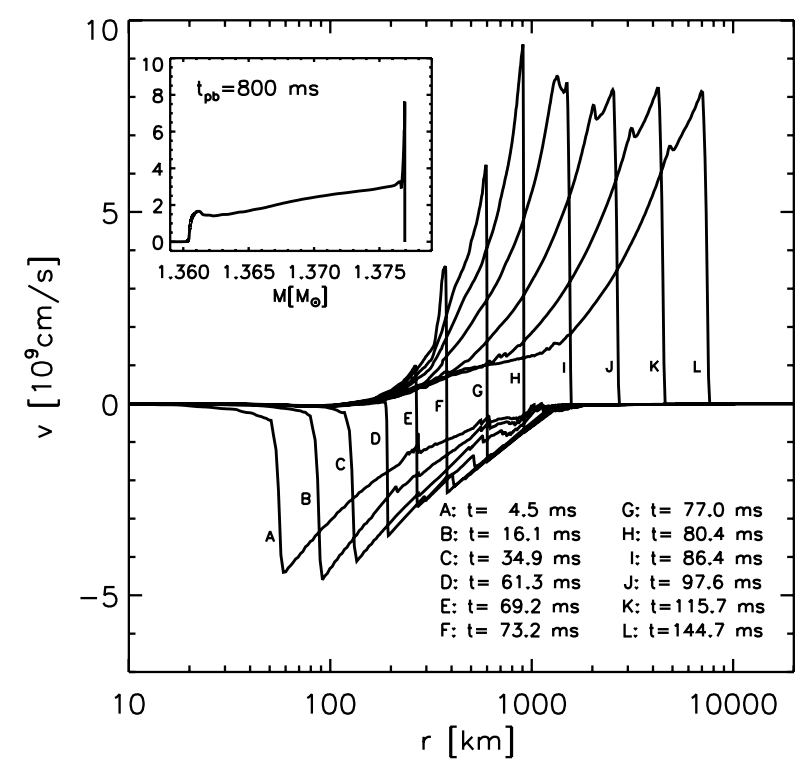

Fig. 3. Velocity profiles as functions of radius for different postbounce times for the simulation with the W\&H EoS. The insert shows the velocity profile vs. enclosed mass at the end of our simulation.

The subsequent expansion of the shock is supported by a combination of different effects. Initially very high mass accretion rates cause the material to pile up between neutrinosphere and shock as it is also observed in the early post-bounce accretion phase of more massive progenitor stars (see, for example, Buras et al. 2006). Second, the rapid decrease of the mass accretion rate contributes to ensure ongoing expansion, because even for quasi-stationary conditions the accretion shock adjusts to a larger radius for smaller mass accretion rates. Finally, as soon as the shock reaches the outer edge of the $\mathrm{C}-\mathrm{O}$ shell, a very steep density decline leads to an outward acceleration of the shock. The last two aspects are linked to the specific structure of $\mathrm{O}-\mathrm{Ne}-\mathrm{Mg}$ cores and discriminate $\mathrm{SN}$ progenitors with such cores from more massive stars. However, despite the shock expansion the material behind the shock has initially still negative velocities and is accreted onto the forming NS (Figs. 1, 3). Note that when the matter right behind the shock starts to expand with the shock, the gas accreted by the shock is gravitationally bound and remains so in passing through the shock. However, $p \mathrm{~d} V$ work excerted from below and to a minor extent energy input by neutrino heating can convert the accretion into an explosion, accelerating a tiny amount of matter $\left(\ll 10^{-3} M_{\odot}\right)$ to move outward with the shock.

While the shock reaches larger radii, the temperature and density behind the shock decrease. High-energy electron neutrinos and antineutrinos, which stream off from their neutrinospheres (represented by the thick solid, dashed, and dashdotted lines in Fig. 1) begin to deposit energy behind the shock mainly by absorption on nucleons. This leads to the formation of a "gain radius" (thin dashed line in Fig. 1) which separates a layer of neutrino cooling around the neutrinosphere from the energy "gain layer" behind the shock (Bethe \& Wilson 1985).

About $80 \mathrm{~ms}$ after bounce for the L\&S EoS and about $60 \mathrm{~ms}$ for the $\mathrm{W} \& \mathrm{H} \mathrm{EoS}$, the neutrino heating timescale, defined by 


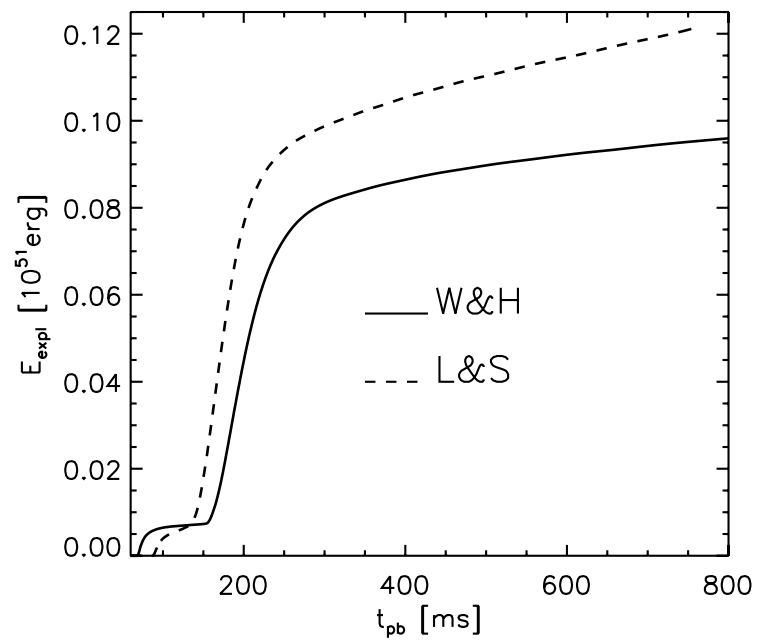

Fig. 4. Explosion energies as functions of time for the simulations with the W\&H and L\&S EoSs. The energy is defined as the volume integral of the total gas energy (internal plus kinetic plus gravitational) in regions where the latter is positive.

the total energy in the gain layer divided by the neutrino heating rate in that region, gets smaller than the advection timescale. The latter is given as the time the accreted matter needs for being advected from the shock to the gain radius. Since the increasing shock radius leads to smaller and smaller postshock velocities, the duration of the deposition of energy via neutrino absorption in the shocked matter increases. The continuous input of energy raises the total energy of the gain layer to a value near zero within roughly $100 \mathrm{~ms}$, unbinding the matter in the gravitational field of the forming NS. The fluid velocity in the layer close to the gain radius therefore starts to become positive (see Figs. 1, 3) and the explosion energy shows a rapid increase (Fig. 4).

The cooling region then becomes more and more narrow as the gain radius retreats towards the neutrinosphere, so that neutrinos diffusing out of the contracting protoneutron star begin to heat the layers right above the neutrinosphere. Gas is thus ablated from the NS surface, and the so-called neutrino-driven wind phase sets in at $t \gtrsim 200 \mathrm{~ms}$ after bounce. Several of the mass shells depicted in Fig. 1 clearly show this process.

At this time the energy in the expanding postshock matter has increased to about $0.1 \times 10^{51} \mathrm{erg}$, rising further due to the power input by the neutrino-driven wind. We extrapolate that the final energy of the explosion will be slightly larger than $0.1 \times 10^{51} \mathrm{erg}$ for the calculation with the $\mathrm{W} \& \mathrm{H} \mathrm{EoS}$ and might be about $50 \%$ higher in case of the L\&S EoS. This is roughly a factor of 10 lower than the canonical $\mathrm{SN}$ value, in contrast to the findings in previous explosion models of $\mathrm{O}-\mathrm{Ne}-\mathrm{Mg}$ cores (Hillebrandt et al. 1984; Mayle \& Wilson 1988).

At the end of our simulation the mass cut, and therefore the baryon mass of the protoneutron star, is around $1.360 M_{\odot}$ for the W\&S EoS (Fig. 1) and about $1.363 M_{\odot}$ for the L\&S EoS. The NS mass will only slightly decrease further because of the ongoing mass loss in the neutrino-driven wind. The mass of the ejecta lies therefore between 0.014 and $0.017 M_{\odot}$. The ejected gas has an electron fraction, $Y_{\mathrm{e}}$, between 0.46 and 0.53

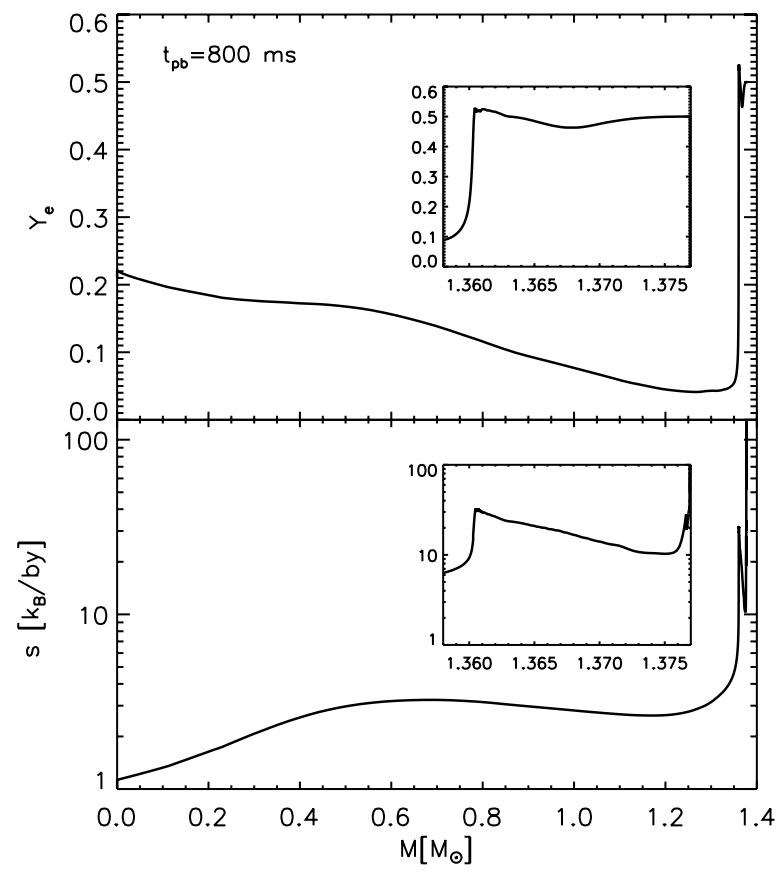

Fig. 5. Profiles of the electron fraction $Y_{\mathrm{e}}$ and entropy $s$ as functions of enclosed mass at the end of our simulation with the W\&H EoS.

and entropy values between 10 and $40 k_{\mathrm{B}}$ per nucleon for both EoSs (see Fig. 5 for the case with the W\&H EoS). Since only about one third of the ejected matter has a $Y_{\mathrm{e}}$ value very close to 0.5 , the mass of ejected ${ }^{56} \mathrm{Ni}$ is certainly smaller than $\sim 0.015 M_{\odot}$. The $Y_{\mathrm{e}}$ values in our models are higher than those in previous simulations of SNe from O-Ne-Mg cores (Mayle \& Wilson 1988). This points to important differences in the neutrino treatment. Spectral Boltzmann transport calculations have recently found early ejecta with $Y_{\mathrm{e}}$ around 0.5 and higher also in (artificial) explosions of more massive progenitors (Buras et al. 2006; Fröhlich et al. 2006). The reason for this difference compared to the older models is a refined description of neutrino spectra formation and in particular of charged-current neutrino-nucleon interactions, including the weak magnetism corrections that were pointed out to be relevant by Horowitz (2002).

\section{Conclusions}

Our 1D simulations of SN explosions from the collapse of $\mathrm{O}-\mathrm{Ne}-\mathrm{Mg}$ cores suggest that such $\mathrm{SNe}$ are powered by neutrino heating and by the neutrino-driven wind of the newly formed NS, similar to what Woosley \& Baron (1992) found in case of the accretion-induced collapse of white dwarfs to NSs. Such events have a low explosion energy $\left(\sim 0.1 \times 10^{51} \mathrm{erg}\right)$ and produce little ${ }^{56} \mathrm{Ni}\left(\$ 10^{-2} M_{\odot}\right)$. Most of the ejecta expand initially with velocities of $2-4 \times 10^{4} \mathrm{~km} \mathrm{~s}^{-1}$, a small fraction has nearly $10^{5} \mathrm{~km} \mathrm{~s}^{-1}$. This is significantly faster than in $\mathrm{SNe}$ of more massive progenitors. Of course, sweeping up the matter of the stellar mantle and envelope, the shock will decelerate, and the ejecta velocities after shock breakout from the stellar surface will be correspondingly lower. During the first second of the explosion, the ejected matter has $0.46 \lesssim Y_{\mathrm{e}} \lesssim 0.53$ and modest 
entropies $\left(10 \lesssim s /\left(k_{\mathrm{B}} /\right.\right.$ by $\left.) \lesssim 40\right)$. Such conditions exclude that r-process elements are formed in this matter during this early phase of the explosion of O-Ne-Mg cores: $Y_{\mathrm{e}}$ is too large for the "classical" low-entropy r-process and $s$ is too low for highentropy r-processing. The ejecta in our models, however, do not show the vast overproduction of some very neutron-rich, rare isotopes like ${ }^{87} \mathrm{Kr}$, which was made in low- $Y_{\mathrm{e}}$ material $\left(Y_{\mathrm{e}} \lesssim 0.44\right)$ in previous simulations (Mayle \& Wilson 1988), and which was interpreted as a severe constraint to the rate of such events. Our models yield considerably less energetic explosions than previous simulations and show other significant differences in the dynamics and explosion characteristics. These are probably mainly linked to the improved treatment of neutrino transport and neutrino-matter interactions.

The small explosion energy obtained in our simulations is more consistent than previous explosion models of O-Ne-Mg cores with the low present expansion velocities $\left(\sim 1500 \mathrm{~km} \mathrm{~s}^{-1}\right)$ of the filaments of the Crab remnant of SN 1054 (Davidson $\&$ Fesen 1985), corresponding to a low kinetic energy of 0.6$1.5 \times 10^{50} \mathrm{erg}$ for an ejecta mass of $4.6 \pm 1.8 M_{\odot}$ in ionized and neutral gas (Fesen et al. 1997). The energy could be even somewhat larger if there were several solar masses of material in an undetected, extended halo.

While our simulations are spherically symmetric, we do not expect any qualitative changes in the multi-dimensional case, and probably only a modest increase of the explosion energy. Since very fast outflow develops on a relatively short timescale after core bounce, nonradial hydrodynamic instabilities are unlikely to have time to merge and grow to very large structures or global asymmetry before the anisotropic pattern freezes out in the accelerating expansion (Scheck et al. 2006). Therefore the recoil velocity of the NS due to anisotropic mass ejection should remain fairly small (see Scheck et al. 2004), in agreement with speculations by Podsiadlowski et al. (2004). Corresponding 2D simulations are in progress.

Our models have also an important bearing on the nuclear EoS constraints deduced by Podsiadlowski et al. (2005) from the low-mass Pulsar B of the double pulsar J0737-3039, which has a gravitational mass of $M_{\mathrm{G}}=1.249 \pm 0.001 M_{\odot}$. Provided the progenitor model we use is valid, the mass loss of the collapsing O-Ne-Mg core during the explosion leaves the neutron star with a baryonic mass of $M_{0}=1.36 \pm 0.002 M_{\odot}$. The error range accounts approximately for variations associated with the employed EoS and the wind ablation after our simulations are terminated. Our value implies a systematic left shift and reduction of the "acceptance rectangle" in Fig. 3 of Podsiadlowski et al. (2005). Combined with the recent measurement of a pulsar of $2.1 \pm 0.2 M_{\odot}$ in PSR J0751+1807 (Nice et al. 2005), which is the largest well determined NS mass so far, this lends viability only to a limited number of NS EoSs which allow for a sufficiently large maximum mass and whose $M_{\mathrm{G}} / M_{0}$-curves pass through the acceptance rectangle.

Based on our findings one might speculate that (B-) stars around $9 M_{\odot}$ are also the progenitors of some of the subluminous type II-P supernovae mentioned in the introduction. In fact, their peculiarities would be explained in a very natural way. The low peak luminosity and extended plateau phase could result from the combination of a low hydrogen envelope mass $\left(\simeq 6 M_{\odot}\right)$ with low expansion velocities $\left(\$ 3000 \mathrm{~km} \mathrm{~s}^{-1}\right)$. The small mass of radioactive ${ }^{56} \mathrm{Ni}$ would explain the low tailluminosity of these objects. An alternative interpretation of subluminous type II-P supernovae is the explosion of rather massive stars with extended envelopes, but otherwise more "normal" explosion energies. This connection is supported by the long duration of the plateau phase of many of these events (cf. Pastorello et al. 2004, and references therein). Provided the observations cover the full duration of the plateau, SN 1997D and $2003 \mathrm{gd}$ may still be viable cases for explosions of stars with main sequence masses around $9 M_{\odot}$ (Hendry et al. 2005). There is a clear difference between such stars and more massive supernova progenitors. The former eject very little amounts (some $10^{-3} M_{\odot}$ ?) of oxygen only, like SN 1997D (Chugai \& Utrobin 2000), whereas the latter produce up to a solar mass or more. Therefore one might be able to distinguish between the two scenarios on the basis of observations by measuring the oxygen lines in the late nebular spectra.

Acknowledgements. We are grateful to K. Nomoto for providing us with the initial data and for encouraging discussions. We thank R. Buras and M. Rampp for their input to this work, A. Marek especially for the W\&H nuclear EoS, and P. Mazzali, A. Pastorello, D. Sauer and V. Utrobin for very helpful discussions about SN observations. This work was supported by the Sonderforschungsbereich 375 "Astroparticle Physics" of the Deutsche Forschungsgemeinschaft and by the International Max Planck Research School (IMPRS). Supercomputer time at the Rechenzentrum Garching is acknowledged.

\section{References}

Arnett, W. D. 1975, ApJ, 195, 727

Baron, E., Cooperstein, J., \& Kahana, S. 1987, ApJ, 320, 300

Bethe, H. A., \& Wilson, J. R. 1985, ApJ, 295, 14

Buras, R., Rampp, M., Janka, H.-T., \& Kifonidis, K. 2006, A\&A, 447, 1049

Burrows, A., \& Lattimer, J. 1985, ApJ, 299, L19

Chugai, N. N., \& Utrobin, V. P. 2000, A\&A, 354, 557

Davidson, K., \& Fesen, R. A. 1985, ARA\&A, 23, 119

Davidson, K., Gull, T. R., Maran, S. P., et al. 1982, ApJ, 253, 696

Fesen, R. A., Shull, J. M., \& Hull, A. P. 1997, AJ, 113, 354

Fröhlich, C., Hauser, P., Liebendoerfer, M., et al. 2006, ApJ, 637, 415

Fryer, C. L., Benz, W., Herant, M., \& Colgate, S. 1999, ApJ, 516, 892

Gott, J. R., Gunn, J. E., \& Ostriker, J. P. 1970, ApJ, 160, L91

Hamuy, M. 2001, ApJ, 582, 905

Hartmann, D., Woosley, S. E., \& El Eid, M. F. 1985, ApJ, 297, 837

Hashimoto, M., Iwamoto, K., \& Nomoto, K. 1993, ApJ, 414, L105

Hendry, M. A., Smartt, S. J., Maund, J. R., et al. 2005, MNRAS, 359, 906

Hillebrandt, W. 1978, Space Sci. Rev., 21, 639

Hillebrandt, W. 1982, A\&A, 110, L3

Hillebrandt, W., Nomoto, K., \& Wolff, R. G. 1984, A\&A, 133, 175

Horowitz, C. J. 2002, Phys. Rev. D, 65, 043001

Janka, H.-T. 1999, unpublished

Langanke, K., Martinez-Pinedo, G., Sampaio, J. M., et al. 2003, Phys. Rev. Lett., 90, 241102

Lattimer, J., \& Swesty, F. 1991, Nucl. Phys. A, 535, 331

Marek, A., Dimmelmeier, H., Janka, H.-T., Müller, E., \& Buras, R. 2006, A\&A, 445, 273

Mayle, R., \& Wilson, J. R. 1988, ApJ, 334, 909 
Miyaji, S., \& Nomoto, K. 1987, ApJ, 318, 307

Miyaji, S., Nomoto, K., Yokoi, K., \& Sugimoto, D. 1980, Astron. Soc. Jap., 32, 303

Nice, D. J., Splaver, E. M., Stairs, I. H., et al. 2005, ApJ, submitted [arXiv: astro-ph/0508050]

Nomoto, K. 1983, Int. Astron. U., 101, 139N

Nomoto, K. 1984, ApJ, 277, 791

Nomoto, K. 1987, ApJ, 322, 206

Nomoto, K., Sparks, W. M., Fesen, R. A., et al. 1982, Nature, 299, 803

Pastorello, A., Zampieri, L., Turatto, M., et al. 2004, MNRAS, 347, 74

Pastorello, A., et al. 2005, MNRAS, submitted

Podsiadlowski, Ph., Langer, N., Poelarends, A. J. T., et al. 2004, ApJ, 612,1044

Podsiadlowski, Ph., Dewi, J. D. M., Poelarends, P., et al. 2005, MNRAS, 361, 1243
Rampp, M., \& Janka, H.-T. 2002, A\&A, 396, 361

Scheck, L., Plewa, T., Janka, H.-T., et al. 2004, PRL, 92, 011103

Scheck, L., Kifonidis, K., Janka, H.-T., \& Müller, E. 2006, A\&A, submitted [arXiv: astro-ph/0601302]

Sumiyoshi, K., Terasawa, M., Mathews, G. J., et al. 2001, ApJ, 562, 880

Takahara, M., Hino, T., Oda, T., et al. 1989, Nucl. Phys. A, 504, 167

Wanajo, S., Tamamura, M., Naoki, I., et al. 2003, ApJ, 593, 968

Wheeler, J. C., Cowan, J. J., \& Hillebrandt, W. 1997, ApJ, 493, L101

Woosley, S. E., \& Baron, E. 1992, ApJ, 391, 228

Woosley, S. E., Weaver, T. A., \& Taam, R. 1980, in type I Supernovae, ed. J. C. Wheeler (Austin: University of Texas), 96

Zampieri, L., Pastorello, A., Turatto, M., et al. 2003, MNRAS, 338, 711 